\section{Hand-carried ultrasound determines volume status of hemodialysis outpatients}

Crucial to minimization of adverse events during hemodialysis, clinical estimation of intravascular volume generally relies on dry weight, which is poorly predictive. Ultrasound assessment of indexed inferior vena cava diameter (VCDi) and inferior vena cava collapsibility index (IVCCI) is a promising alternative to dry weight estimation, but usually requires expensive equipment and highly skilled operators.

Brennan et al. enrolled 89 patients from two dialysis clinics in a study to examine the practicability of non-cardiologist operators using inexpensive, portable ultrasound devices to assess VCDi and IVCCl. Assessments were performed immediately before hemodialysis and within $30 \mathrm{~min}$ of session completion. High-quality images were obtained in $89 \%$ of patients. Ultrasound yielded estimates of intravascular volume that were significantly different to those based on dry weight; although $89 \%$ of patients were initially above their established dry weight, $39 \%$ and $47 \%$ of these patients were hypovolemic according to IVCCI and VCDi measurements, respectively.

Predialysis hypovolemia as assessed by VCDi was correlated with an increased incidence of chest pain or cramping during treatment $(P=0.06)$. VCDi measurements taken during intradialytic events revealed $92 \%$ of subjects $(n=11)$ to be hypovolemic. The four medical residents that performed the ultrasound measurements, and their attending nephrologists, were satisfied with the technique and reported minimal disruption to patient care.

The authors conclude that the use of handcarried ultrasound in outpatient hemodialysis clinics is a viable means of assessing intravascular volume status for prediction of adverse intradialytic events.

Original article Brennan JM et al. (2006) Handcarried ultrasound measurement of the inferior vena cava for assessment of intravascular volume status in the outpatient hemodialysis clinic. Clin J Am Soc Nephrol 1: 749-753

\section{Mycophenolate mofetil treatment for acute interstitial nephritis}

Acute interstitial nephritis (AIN) is often caused by an allergic reaction to a medication; it can also be associated with autoimmune conditions and with various infections. Most causes of AIN seem to respond to immunosuppressants such as corticosteroids, but some patients are resistant to or intolerant of corticosteroid therapy. Preddie et al. have investigated whether the immunosuppressant mycophenolate mofetil (MMF) could be useful in such patients.

The study included eight patients with biopsyproven AIN who had received $\geq 6$ months of corticosteroid treatment but had suffered relapses, intolerance or resistance to corticosteroid therapy. Patients received twice-daily MMF doses ranging from $500 \mathrm{mg}$ to $1,000 \mathrm{mg}$.

After a mean of 28 months of follow-up (range 14-40 months, with a mean of 24 months of MMF treatment), mean serum creatinine level decreased from $2.3 \mathrm{mg} / \mathrm{dl}$ to $1.6 \mathrm{mg} / \mathrm{dl}$ $(203 \mu \mathrm{mol} / /$ to $141 \mu \mathrm{mol} / \mathrm{l})$. Six of eight patients showed improvement in renal function-defined as a decrease in serum creatinine level of $\geq 0.3 \mathrm{mg} / \mathrm{dl}(26.5 \mu \mathrm{mol} / \mathrm{l})$-following MMF treatment. Serum creatinine levels remained stable in the other two patients. At their final followup visit, all patients had discontinued corticosteroids, and five patients had discontinued MMF therapy. One patient had a myocardial infarction during MMF therapy and died of cardiac disease, but this was thought to be unrelated to MMF. No serious adverse effects of MMF were reported.

Although further studies are needed, MMF appears to be a useful alternative treatment for patients with steroid-resistant or steroidintolerant AIN, and might be a good first-line option in patients with conditions such as obesity or diabetes, for whom steroid treatment is undesirable.

Original article Preddie DC et al. (2006) Mycophenolate mofetil for the treatment of interstitial nephritis. Clin J Am Soc Nephrol 1: 718-722 